\title{
Análise do estado funcional e força muscular de adultos e idosos em Unidade de Terapia Intensiva: Coorte prospectiva
}

\author{
Analysis of functional status and muscle strength in adults and \\ older adults in an intensive care unit: a prospective cohort study
}

Gabriela Sousa Martins (https://orcid.org/0000-0001-5478-4152) ${ }^{1}$

Samara Vasconcelos Toledo (https://orcid.org/0000-0003-1922-6643) ${ }^{2}$

Joanlise Marco de Leon Andrade (https://orcid.org/0000-0002-3984-3799) ${ }^{3}$

Eduardo Yoshio Nakano (https://orcid.org/0000-0002-9071-8512) ${ }^{3}$

Renato Valduga (https://orcid.org/0000-0002-7006-8145) ${ }^{4}$

Leonardo Petrus da Silva Paz (https://orcid.org/0000-0003-2926-4547) ${ }^{1}$

Gerson Cipriano Júnior (https://orcid.org/0000-0001-6323-7003) ${ }^{1}$

Graziella França Bernardelli Cipriano (https://orcid.org/0000-0001-9560-6520) ${ }^{2}$
${ }^{1}$ Programa de Pós-

Graduação em Ciências

e Tecnologias em Saúde,

Universidade de Brasília.

Centro Metropolitano

conjunto A lote $011^{\circ}$

andar sala A1-04/67.

72220-275 Brasília DF

Brasil.

gabrielamartins.unb@

gmail.com

${ }^{2}$ Programa de Pós-

Graduação em Ciências da

Reabilitação, Universidade

de Brasília. Brasília DF

Brasil.

${ }^{3}$ Departamento de

Estatística, Universidade de

Brasília. Brasília DF Brasil.

${ }^{4}$ Secretaria de Estado de

Saúde, Distrito Federal.

Brasília DF Brasil.

\begin{abstract}
The aim of this study was to analyze and assess the association between functional status (FS) and muscle strength (MS) in young adults, adults and older adults in an intensive care unit (ICU). We conducted a prospective cohort study with 48 patients. FS was assessed using the Functional Status Score for the Intensive Care Unit (FSS-ICU) and MS was measured using the Medical Research Council Sum-Score (MRCSS) and by testing handgrip strength (HS). The assessments were performed on awakening and ICU discharge. The data were analyzed using the Kruskall-Wallis, chi-squared, Wilcoxon and Spearman's correlation tests. FS and MRC-SS scores were higher on ICU discharge in all groups. Gains were lowest in the older adult group. HS was greater in both hands on ICU discharge in all groups except the adults. FSS-ICU on both awakening and ICU discharge was highest in the adults; HS-R was lowest in the older adults. There was a strong association between FS and MS in the young adults and adults. FS and MS showed progressive improvement during ICU stay. Gains in FS and MS on awakening and ICU discharge were lowest among the older adults. Important associations were found between these variables in all groups except the older adults. This can be explained by the multifactorial nature of illness and incapacity in this group.
\end{abstract}

Key words Health assessment, Intensive care unit, Physical function, Muscle strength, Older persons
Resumo Avaliar e correlacionar o estado funcional (EF) e a força muscular (FM) em adultos jovens, adultos e idosos em Unidade de Terapia Intensiva (UTI). Coorte prospectiva com 48 pacientes avaliando EF por meio do Functional Status Score for the Intensive Care Unit (FSS-ICU) e FM pelo Medical Research Council Sum-Score (MRC-SS) e força de preensão palmar (FPP), no despertar e na alta da UTI. Dados analisados pelos testes Kruskall-Wallis, Qui-quadrado, Wilcoxon e Correlação de Sperman. Na comparação do despertar em relação à alta, o EF e o MRC-SS foram maiores na alta em todos os grupos, com menor ganho nos idosos. A FPP aumentou bilateralmente na alta, exceto no grupo de adultos, pois apresentavam valores maiores no despertar. Na comparação dos grupos, o FSS-ICU foi maior nos adultos no despertar e alta, e a FPP-D menor nos idosos. $O E F$ apresentou forte associação com a FM em adultos jovens e adultos, pois ambos apresentam progressiva melhora durante a permanência na UTI. Os idosos apresentam menor ganho do estado funcional, além de apresentarem menor força muscular desde o despertar. Estas variáveis apresentam importante associação apenas nos adultos jovens e adultos, considerando a natureza multifatorial do processo de adoecimento $e$ incapacidade nos idosos.

Palavras-chave Avaliação em saúde, Unidade de Terapia Intensiva, Funcionalidade, Força muscular, Idoso 


\section{Introdução}

O avanço tecnológico e o tratamento interdisciplinar na Unidade de Terapia Intensiva (UTI) tem proporcionado o aumentado da sobrevida dos pacientes críticos. Notoriamente, esses pacientes estão sujeitos ao repouso prolongado no leito, proporcionando um declínio da capacidade funcional, força muscular e função cognitiva ${ }^{1-3}$. Nos idosos essas alterações podem ocorrer de forma acentuada, em decorrência das limitações físicas e mentais durante o processo de envelhecimento, sendo este dinâmico e multifatorial ${ }^{4,5}$.

Durante a hospitalização, o imobilismo favorece a perda de massa muscular de forma precoce e rápida nos pacientes críticos de diferentes faixas etárias, promovendo redução de fibras musculares do tipo II, propriedades contráteis, aumento das citocinas inflamatórias, e proteólise muscular ${ }^{5-9}$. Estas alterações são responsáveis por um declínio de aproximadamente $40 \%$ da força muscular na primeira semana de imobilização em UTI, o que favorece o surgimento da fraqueza muscular adquirida na UTI (FMA-UTI) ${ }^{6,7}$.

A persistência da fraqueza muscular está associada à redução da capacidade aeróbica, impactando na qualidade de vida e nas atividades de vida diária ${ }^{2}$, além de propiciar aumento dos números de re-hospitalizações e mortalidade ${ }^{10-13}$. Recente revisão sistemática ${ }^{3}$ destacou que a avaliação da capacidade funcional e da força muscular durante a permanência na UTI é de extrema relevância, devendo ocorrer precocemente, para identificar pacientes com potencial risco de redução física e funcional.

Diversos estudos ${ }^{10,13-19}$ evidenciam as alterações da capacidade funcional dos doentes críticos, entretanto não descrevem as alterações da capacidade físico-funcional de acordo com a faixas etárias. Esta informação é de extrema relevância, haja vista a ampla faixa etária de pacientes admitidos nas unidades de terapia intensiva, a fim de otimizar os planos de ações terapêuticas, para que sejam iniciados de forma mais precoce e individualizada, incluindo a UTI como a primeira fase do processo de reabilitação ${ }^{12}$. Assim, o presente estudo tem por objetivo analisar o estado funcional de adultos jovens, adultos e idosos no momento do despertar até a alta da UTI e suas associações com as medidas de força muscular.

\section{Métodos}

\section{Características do estudo}

Trata-se de um estudo de coorte prospectivo, realizado de acordo com as recomendações do Strengthening the Reporting of Observational Studies in Epidemiology (STROBE) guidelines ${ }^{20}$. O estudo foi realizado na UTI adulto de um Hospital público na cidade de Ceilândia, Brasília, Distrito Federal (Hospital Regional de Ceilândia - HRC) no período de março de 2015 a julho de 2016.

Este estudo foi aprovado pelo Comitê de Ética e Pesquisa em Seres Humanos da Fundação de Ensino e Pesquisa em Ciências da Saúde, Secretaria de Saúde do Distrito Federal. Todos os sujeitos e ou/ responsáveis legais foram informados do protocolo de estudo e concordaram com sua participação, a partir da assinatura do Termo de Consentimento, Livre e Esclarecido (TCLE).

\section{Participantes}

A amostra foi recrutada por meio de técnica de amostragem não-probabilística, do tipo conveniência com pacientes críticos internados. Foram elegíveis os pacientes com idade $\geq 18$ anos, de ambos os sexos, com nível de consciência superior a 8 , de acordo com a escala de coma de Glasgow.

Foram excluídos os participantes que apresentaram fraturas instáveis, sequelas motoras e ou/neurológicas prévias à internação, cicatrizes e/ou outras condições que inviabilizassem a sedestação, e pacientes em medidas de cuidados paliativos.

\section{Protocolo de avaliação}

Utilizou-se o sistema eletrônico de prontuários para a obtenção dos dados clínicos durante a permanência na UTI. As variáveis analisadas foram sexo, idade, comorbidades prévias à internação, diagnóstico de admissão na UTI, relato clínico de sepse, índice de massa corporal (IMC), índice de gravidade da doença medido pelo Acute Physiology and Chronic Health Classification System II (APACHE II), medicamentos (tempo de uso de corticoides, sedativos e drogas vasoativas), dias em ventilação mecânica (VM) e o tempo de internação (pré-UTI e durante a permanência na UTI). 
Todos os participantes foram submetidos a uma avaliação do estado funcional e força muscular, no momento do despertar e na alta da UTI, e posteriormente separados em três grupos distintos, sendo adultos jovens, adultos e idosos. $\mathrm{O}$ despertar foi definido como o primeiro dia em que os indivíduos responderam no mínimo três dos cinco critérios de De Jonghe et al. ${ }^{16}$ : Abra e feche os olhos, olhe para mim, abra a boca e coloque a lingua para fora, balance a cabeça e levante as sobrancelhas enquanto conto até cinco.

As avaliações foram realizadas pela equipe de pesquisadores, composta por fisioterapeutas previamente treinados e capacitados para aplicação das escalas e instrumentos de avaliação. A UTI possuía assistência de fisioterapia $24 \mathrm{~h}$ por dia, e todos os pacientes recebiam no mínimo duas sessões diárias de fisioterapia, de acordo com o protocolo do setor. $\mathrm{O}$ estado funcional foi avaliado por meio da escala Functional Status Score for the Intensive Care Unit (FSS-ICU), que consiste em uma medida que avalia a capacidade dos pacientes em realizar tarefas à beira leito. O FSS-ICU é uma medida desenvolvida para o ambiente de UTI, traduzido e adaptado para a população brasileira que avalia cinco tarefas, quais sejam: 1) rolar, 2) transferência de supino para sentado, 3) sentado na beira do leito, 4) a transferência do sentado para do de pé e 5) deambular ${ }^{21,22}$.

Os participantes foram orientados a realizar as tarefas no leito de forma mais independente possível e caso não conseguissem, a assistência era oferecida pelo avaliador. Ao final da avaliação foi atribuída uma pontuação conforme o nível de assistência requisitado para cada sujeito. A pontuação era constituída por um sistema de 8 pontos para as cinco tarefas, sendo zero atribuído àqueles incapazes de executar as atividades, e 7 àqueles completamente independente, perfazendo um escore total máximo de 35 pontos, em quanto maior a pontuação, melhor o $\mathrm{EF}^{21}$.

Na sequência, a avaliação da força muscular foi realizada a partir da escala Medical Research Council Sum-Score (MRC-SS) e da força de preensão palmar (FPP) por meio da dinamometria. O MRC-SS avalia seis grupos musculares: abdutores de ombro, flexores de cotovelo, extensores de punho, flexores de quadril, extensores de joelho e os dorsiflexores do tornozelo bilateralmen$\mathrm{te}^{17,18}$.

O teste do MRC-SS foi realizado inicialmente com o membro do paciente posicionado contra a gravidade, demonstrando passivamente o movimento que seria avaliado. Em seguida o pacien- te era instruído a realizar o movimento contra a gravidade e, caso não fosse capaz de realizar o movimento, a posição era modificada, com objetivo de reduzir a carga ${ }^{23}$. Uma resistência isométrica foi aplicada ao final do arco do movimento para testar cada grau de força muscular, conforme descrito por Parry ${ }^{18}$. Cada grupo muscular recebeu uma pontuação que variou de 0 (paralisia total) a 5 (força muscular normal), perfazendo um score com variação de 0 a 60 pontos ${ }^{17}$. O score final menor do que 48 pontos na avaliação foi utilizado para diagnóstico de Fraqueza Muscular Adquirida na UTI (FMA-UTI) ${ }^{24}$.

A força muscular foi avaliada pela força de pressão palmar (FPP), por meio do dinamômetro digital (Jamar Plus ${ }^{\circledast}$, Patterson Medical Ltda., Illinois, EUA). Todos os participantes foram posicionados sentados, com cotovelo a $90^{\circ}$ de flexão e antebraços em posição neutra. Em seguida, os pacientes foram orientados a pressionarem o equipamento realizando o máximo de força possível. Foi ofertado um incentivo verbal durante o teste e o equipamento foi apoiado pelo avaliador, sendo realizado ao todo três manobras bilateralmente, obtendo o valor médio das medidas para análises $^{19,24}$.

\section{Análise estatística}

Os dados categóricos foram apresentados em frequência total (f) e relativa (\%), e os dados contínuos em mediana e intervalo interquartil, considerando a normalidade dos dados, avaliada por meio do teste de Kolmogorov-Smirnov. Todos os testes consideraram hipóteses bilaterais e um nível de significância de 5\% $(\mathrm{a}=0.05)$.

A amostra geral foi categorizada em três grupos etários: adultos jovens ( $\geq 18$ até 39 anos), adultos ( $\geq 40$ até 59 anos) e idosos ( $\geq 60$ anos). Para comparações dos grupos em relação às características clínicas, estado funcional e força muscular no momento do despertar e na alta da UTI foi utilizado o Teste Kruskall Wallis com pós-teste de Muller-Dunn para variáveis numéricas e o Teste Qui-Quadrado para comparações das variáveis categóricas entre os grupos.

Para análise do estado funcional e da força muscular entre o despertar e a alta da UTI foi utilizado o teste de Wilcoxon. A correlação das medidas de FSS-SS com o MRC-SS e a FPP no despertar e alta da UTI foram realizadas por meio do teste de correlação de Spearman.

As análises foram realizadas por meio dos pacotes estatísticos Graphpad Prism 5 for MAC e SPSS V.24 for MAC. 


\section{Tamanho amostral}

O cálculo do tamanho da amostra se baseou na evolução das medidas de FSS-SS e MRC-SS entre o despertar e alta da UTI. Considerou-se uma diferença clinicamente significativa de meio desvio-padrão ( tamanho de efeito $=0,5)$, nível de significância de $95 \%$, poder de $80 \%$ e um percentual de desistências (dropout) de 20\%. Sob essas condições, a amostra mínima foi calculada em 40 pacientes.

\section{Resultados}

Dentre os 54 pacientes elegíveis, 48 realizaram avaliação do EF e FM no momento do despertar e alta da UTI, dos quais 16 eram adultos jovens, 17 adultos e 15 idosos. A amostra geral apresentou uma idade mediana (IIQ) de 47 (36-62) anos e foi predominantemente composta do gênero masculino (62\%), sendo as doenças respiratórias a principal causa de admissão na UTI (27\%) (Figura 1).

Os idosos apresentaram pontuação maior de acordo com o APACHE II comparado aos adultos e adultos jovens [25(19-29) vs 17(14-24) vs 16(14-19); $\mathrm{p}=0,077]$ e a principal comorbidade foi a Hipertensão Arterial (100\%). O uso de suporte ventilatório invasivo foram semelhantes entre os grupos $75 \%$, $71 \%$ e $80 \%(p=0,828)$ e o mesmo ocorreu com o tempo de permanência na UTI [9(5-21) vs 11(7-20) vs 12(8-21); $\mathrm{p}=0,653]$, respectivamente.

Em relação ao tempo em VM o período foi similar entre o grupos, [5 (0-12) vs 6 (0-11) vs 7 (31-12); $\mathrm{p}=0,824)]$. A incidência de FMA-UTI no despertar foi maior nos idosos, acometendo 93\% da amostra enquanto a incidência nos adultos jovens e adultos foi de $50 \%$ e $59 \%(p=0,026)$ (Tabela 1).

$\mathrm{Na}$ comparação do estado funcional e força muscular da amostra geral, entre os momentos do despertar e da alta de UTI, observou-se diferenças significativas para as variáveis FSS-ICU [15(7-24) vs 26(16-32); $\mathrm{p}=0,001)]$, MRC-SS [44(37-53) vs 52(44-56); $\mathrm{p}=0,000)]$, FPP-D [14(11-25) vs 18(11-25); $\mathrm{p}=0,002)]$, e FPP-E [13(8-22) vs 16(10-23); $\mathrm{p}=0,004)$. Essas medidas apresentaram um aumento gradativo no momento da alta (Tabela 2).

Em relação à avaliação do FSS-ICU, observamos um aumento expressivo no momento da alta nas diferentes faixas etárias [17(7-28) vs 21(1034), $\mathrm{p}=0,048 ; 18(9-30)$ vs 32(27-34), $\mathrm{p}=0,001$; 11(6-18) vs 23(13-28), $\mathrm{p}=0,003)]$. O MRC-SS

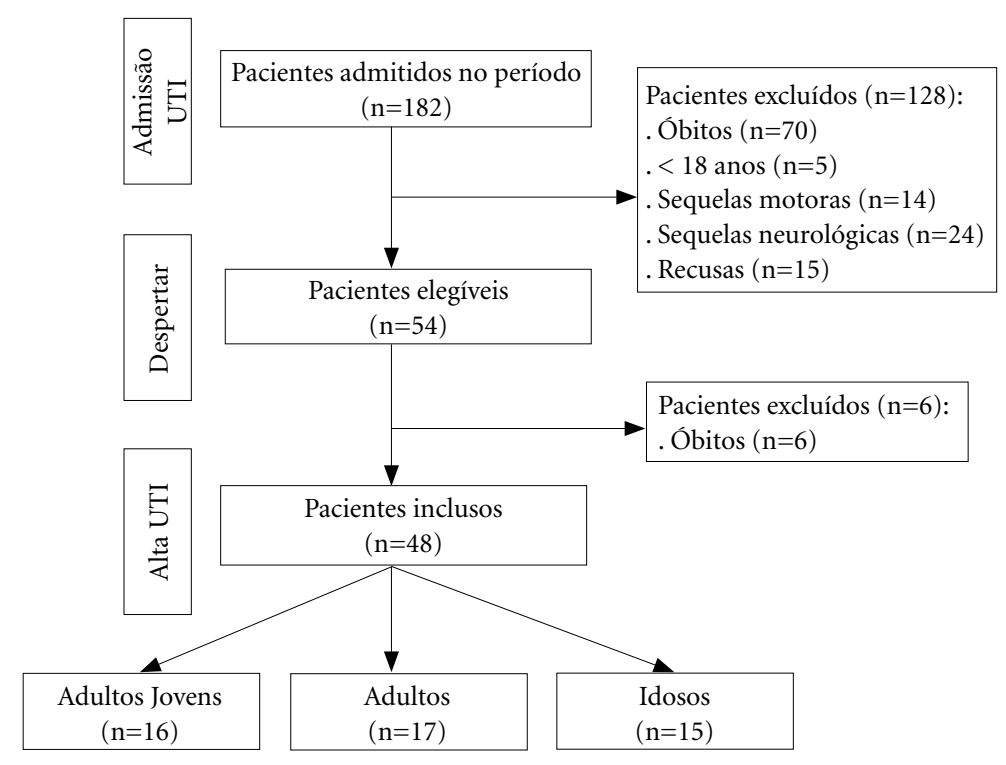

Figura 1. Fluxograma do Estudo de Coorte. 
Tabela 1. Características clínicas e da internação da amostra geral e dos pacientes críticos adultos jovens e idosos, durante a permanência na UTI.

\begin{tabular}{|c|c|c|c|c|c|}
\hline \multirow[t]{2}{*}{ Variáveis } & Geral & $\begin{array}{c}\text { Adultos } \\
\text { Jovens }\end{array}$ & Adultos & Idosos & \multirow[t]{2}{*}{$\mathbf{p}$} \\
\hline & $(n=48)$ & $(n=16)$ & $(n=17)$ & $(n=15)$ & \\
\hline Idade, mediana (IIQ) & $47(36-62)$ & $31(27-36)$ & $47(44-52)$ & $65(62-74)$ & $<0,001$ \\
\hline Sexo, masculino, f (\%) & $30(62)$ & $13(81)$ & $9(53)$ & $8(53)$ & 0,165 \\
\hline APACHE II, mediana (IIQ) & $18(14-27)$ & $16(14-19)$ & $17(14-24)$ & $25(19-29)$ & 0,077 \\
\hline \multicolumn{6}{|l|}{ Comorbidades, $\mathrm{f}(\%)$} \\
\hline HAS & $27(56)$ & $3(19)$ & $9(53)$ & $15(100)$ & $<0,001$ \\
\hline Diabetes Mellitus & $13(27)$ & $3(19)$ & $5(29)$ & $5(33)$ & 0,577 \\
\hline ICC & $7(15)$ & 0 & $2(12)$ & $4(27)$ & 0,330 \\
\hline DPOC & $3(6)$ & 0 & $1(6)$ & $2(13)$ & 0,512 \\
\hline AVC & $4(8)$ & 0 & $1(6)$ & $3(20)$ & 0,199 \\
\hline IRC & $6(12)$ & $1(6)$ & $3(18)$ & $2(13)$ & 0,506 \\
\hline IMC, mediana (IIQ) & $23(22-29)$ & $22(21-28)$ & $24(22-30)$ & $23(22-29)$ & 0,251 \\
\hline \multicolumn{6}{|l|}{ Causas Admissão UTI, f (\%) } \\
\hline Respiratórias & $13(27)$ & $4(25)$ & $6(35)$ & $3(20)$ & 0,07 \\
\hline Cardiovasculares & $10(20)$ & $3(19)$ & $4(23)$ & $3(20)$ & \\
\hline Infecciosas & $8(17)$ & $3(19)$ & $2(12)$ & $3(20)$ & \\
\hline Pós-operatório & $3(6)$ & $1(6)$ & $1(6)$ & $1(7)$ & \\
\hline Neurológicas & $4(8)$ & $2(12)$ & $2(12)$ & $0(0)$ & \\
\hline Inflamatórias & $8(17)$ & $3(19)$ & $1(6)$ & $4(26)$ & \\
\hline Intoxicação & $2(4)$ & 0 & $1(6)$ & $1(7)$ & \\
\hline \multicolumn{6}{|l|}{ Medicamentos, mediana (IIQ) } \\
\hline Dias corticoides & $4(0-9)$ & $7(0-12)$ & $3(0-9)$ & $1(0-7)$ & 0,153 \\
\hline Dias de sedativos & $3(0-7)$ & $4(0-9)$ & $2(0-6)$ & $4(2-9)$ & 0,499 \\
\hline Dias de drogas vasoativas & $3(0-8)$ & $0(0-7)$ & $2(0-8)$ & $5(2-11)$ & 0,204 \\
\hline Sepse, n (\%) & $16(33)$ & $3(19)$ & $6(36)$ & $7(47)$ & 0,251 \\
\hline Uso de VM, $\mathrm{f}(\%)$ & $36(75)$ & $12(75)$ & $12(71)$ & $12(80)$ & 0,828 \\
\hline Dias VM, mediana (IIQ) & $6(2-12)$ & $5(0-12)$ & $6(0-11)$ & $7(3-12)$ & 0,824 \\
\hline Dias pré- UTI, mediana (IIQ) & $2(1-8)$ & $3(1-8)$ & $2(1-8)$ & $3(2-10)$ & 0,509 \\
\hline Dias de internação UTI, mediana (IIQ) & $10(7-20)$ & $9(5-21)$ & $11(7-20)$ & $12(8-21)$ & 0,653 \\
\hline Dias até o despertar, mediana (IIQ) & $7(3-11)$ & $4(3-12)$ & $7(3-13)$ & $8(6-11)$ & 0,439 \\
\hline \multicolumn{6}{|l|}{ FMA-UTI no despertar, f (\%) } \\
\hline MRC-SS $\leq 48$ pontos & $32(67)$ & $8(50)$ & $10(59)$ & $14(93)$ & 0,026 \\
\hline \multicolumn{6}{|l|}{ Fisioterapia, mediana (IIQ) } \\
\hline Motora, total sessões & $19(10-36)$ & $10(6-44)$ & $19(12-36)$ & $21(14-41)$ & 0,499 \\
\hline Respiratória, total sessões & $16(10-38)$ & $12(6-49)$ & $17(11-36)$ & $22(14-41)$ & 0,499 \\
\hline
\end{tabular}

Dados expressos em mediana e intervalo interquartil (IIQ), frequência absoluta (f) e percentual (\%) com p- valor para os testes: Teste Kruskall Wallis com pós teste de Muller-Dunn e Teste Qui-Quadrado, nível de significância $\mathrm{p} \leq 0.05$. APACHE II: Acute Physiology and Chronic Health Classification System II, HAS: Hipertensão Arterial, ICC: Insuficiência Cardíaca Congestiva; DPOC: Doença Pulmonar Obstrutiva Crônica; AVC: Acidente Vascular Cerebral; IRC: Insuficiência Renal Crônica; IMC: Índice de Massa Corporal, UTI: Unidade de Terapia Intensiva, VM: Ventilação Mecânica, FMA-UTI: Fraqueza Muscular Adquirida na UTI, MRC-SS: Medical Research Council Sum-Score.

Fonte: Elaborada pelos autores.

apresentou comportamento semelhante [48(3256) vs 53(36-60), $\mathrm{p}=0,050 ; 46(39-56)$ vs 55(4556), $\mathrm{p}=0,031$; 40(34-46) vs 48(44-52), $\mathrm{p}=0,003)]$. Somente nos adultos a FPP-D e E permaneceram inalteradas $(p=0,211 ; p=0,244)$. O membro di- reto, foi caracterizado dominante em $75 \%$ dos adultos jovens, $71 \%$ dos adultos e $80 \%$ nos idosos. Os dados estão apresentados na Tabela 2.

$\mathrm{Na}$ análise do estado funcional e força muscular entre os grupos adultos jovens, adultos e 
Tabela 2. Avaliação do estado funcional e força muscular entre os momentos do despertar e alta da UTI nos diferentes grupos.

\begin{tabular}{|c|c|c|c|c|c|c|c|c|c|c|c|c|}
\hline \multirow{3}{*}{ Variáveis } & \multirow{2}{*}{\multicolumn{3}{|c|}{$\begin{array}{c}\text { Geral } \\
(n=48)\end{array}$}} & \multirow{2}{*}{\multicolumn{3}{|c|}{$\begin{array}{c}\text { Adultos Jovens } \\
(\mathrm{n}=16)\end{array}$}} & \multirow{2}{*}{\multicolumn{3}{|c|}{$\begin{array}{c}\text { Adultos } \\
(\mathbf{n}=17) \\
\end{array}$}} & \multirow{2}{*}{\multicolumn{3}{|c|}{$\begin{array}{l}\text { Idosos } \\
(n=15) \\
\end{array}$}} \\
\hline & & & & & & & & & & & & \\
\hline & Despertar & Alta & $\mathbf{p}$ & Despertar & Alta & $\mathbf{p}$ & Despertar & Alta & $\mathbf{p}$ & Despertar & Alta & p \\
\hline FSS-ICU & $\begin{array}{r}15 \\
(7-24)\end{array}$ & $\begin{array}{r}26 \\
(16-32)\end{array}$ & 0,001 & $\begin{array}{r}17 \\
(7-28)\end{array}$ & $\begin{array}{r}21 \\
(10-34)\end{array}$ & 0,048 & $\begin{array}{r}18 \\
(9-30)\end{array}$ & $\begin{array}{r}32 \\
(27-34)\end{array}$ & 0,001 & $\begin{array}{r}11 \\
(6-18)\end{array}$ & $\begin{array}{r}23 \\
(13-28)\end{array}$ & 0,002 \\
\hline MR & $\begin{array}{r}44 \\
(37-53)\end{array}$ & $\begin{array}{r}52 \\
(44-56)\end{array}$ & 0,000 & $\begin{array}{r}48 \\
(32-56)\end{array}$ & $\begin{array}{r}53 \\
(36-60)\end{array}$ & 0,050 & $\begin{array}{r}46 \\
(39-56)\end{array}$ & $\begin{array}{r}55 \\
(45-56)\end{array}$ & 0,031 & $\begin{array}{r}40 \\
(34-46)\end{array}$ & $\begin{array}{r}48 \\
(44-52)\end{array}$ & 0,003 \\
\hline FPP-D & $\begin{array}{r}14 \\
(11-25)\end{array}$ & $\begin{array}{r}18 \\
(11-25)\end{array}$ & 0,002 & $\begin{array}{r}17 \\
(7-27)\end{array}$ & $\begin{array}{r}21 \\
(11-27)\end{array}$ & 0,009 & $\begin{array}{r}18 \\
(14-38)\end{array}$ & $\begin{array}{r}18 \\
(13-27)\end{array}$ & 0,211 & $\begin{array}{r}12 \\
(11-14)\end{array}$ & $\begin{array}{r}16 \\
(10-19)\end{array}$ & 0,023 \\
\hline FPP-E & $\begin{array}{r}13 \\
(8-22) \\
\end{array}$ & $\begin{array}{r}16 \\
(10-23)\end{array}$ & 0,004 & $\begin{array}{r}14 \\
(6-28) \\
\end{array}$ & $\begin{array}{r}16 \\
(9-31) \\
\end{array}$ & 0,009 & $\begin{array}{r}18 \\
(10-27)\end{array}$ & $\begin{array}{r}18 \\
(11-24)\end{array}$ & 0,241 & $\begin{array}{r}11 \\
(6-16)\end{array}$ & $\begin{array}{r}12 \\
(9-21)\end{array}$ & 0,019 \\
\hline
\end{tabular}

Dados expressos em mediana (intervalo interquartil). p- valor para teste de Wilcoxon, nível de significância $p \leq 0.05$.

Legenda: FSS-ICU: Functional Status Score for the Intensive Care Unit, MRC-SS: Medical Research Council Sum-Score, FPP: Força de preensão palmar, D: Direita, E: Esquerda.

Fonte: Elaborada pelos autores.

idosos no momento do despertar e na alta da UTI, observamos diferença significativas para o FSS-ICU no despertar [17(7-28) vs 18(9-30) vs $11(6-18) ; \mathrm{p}=0,040]$ e na alta [ $21(10-34) ; 32(27$ 34); 23(13-28); $\mathrm{p}=0,013]$. A FPP-D foi maior no despertar [17(7-27); 18(14-38);12(11-14); $\mathrm{p}=0,050]$ (Figura 2).

$\mathrm{Na}$ avaliação da amostra geral, a FSS-ICU apresentou forte correlação com a MRC- no despertar $(\mathrm{r}=0,74 ; \mathrm{p}=0,001)$ e na alta $(\mathrm{r}=0,75$; $\mathrm{p}=0,001)$. A correlação da FSS-ICU com a FPP -D foi moderada no despertar $(r=0,61 ; p=0,00)$ e na alta $(r=0,57 ; p=0,007)$, e o mesmo comportamento ocorreu na FPP-E no momento do $\operatorname{despertar}(\mathrm{r}=0,57 ; \mathrm{p}=0,001)$ e na alta $(\mathrm{r}=0,50$; $\mathrm{p}=0,001)$.

No despertar, evidenciamos uma correlação forte do FSS-ICU com o MRC no grupo adultos jovens e adultos. A correlação com a FPP-D no grupo adultos jovens foi moderada e no grupo adultos forte e com a FPP-E foi moderada nos dois primeiros grupos. No momento da alta da UTI, observamos uma correlação forte do FSS-ICU com o MRC, no grupo dos adultos jovens e nos adultos e uma correlação moderada com a FPP-D e forte com a FPP-E $(r=0,80 ; p=0,001)$. Os idosos apresentaram uma associação fraca das variáveis do estado funcional e força muscular em ambos os momentos (Figura 3).

\section{Discussão}

O presente estudo identificou uma mudança gradual do comportamento do estado funcional e força muscular nos pacientes críticos. Observamos que os idosos apresentaram um menor ganho de funcionalidade e força muscular, com possível potencial de recuperação dessas medidas. Estas variáveis apresentam importante associação nos adultos jovens e adultos. Esses resultados permitem direcionar ações terapêuticas futuras, conforme as necessidades clínicas de cada faixa etária, indicando que os idosos necessitam de condutas preventivas e direcionadas a fim de minimizar os efeitos deletérios da hospitalização.

A ocorrência do declínio funcional em pacientes críticos, durante a permanência na UTI, independente de faixa etária nos setores de terapia intensiva, tem demonstrado relevante destaque na literatura. As revisões sistemáticas existentes ${ }^{2,25-27}$ destacam uma redução da função dos sistemas cardiovasculares e musculoesqueléticos, que representam potencial risco ao estado de saúde dos indivíduos, devendo portanto ser investigados e identificados precocemente para intervenção em tempo hábil.

Em nosso trabalho, identificamos um predomínio de indivíduos do gênero masculino, similarmente aos encontrados com os estudos internacionais ${ }^{21,28}$ em pacientes críticos em UTIs. Os homens apresentam uma baixa adesão a medidas preventivas e curativistas, pois tendem a cuidar menos da saúde por dificuldades relacionadas ao distanciamento do trabalho e, portanto, buscam serviços de saúde em situações críticas, quando adoecem de modo severo ${ }^{29,30}$.

Além disso, culturalmente os homens desde a infância são ensinados a demonstrarem à socie- 

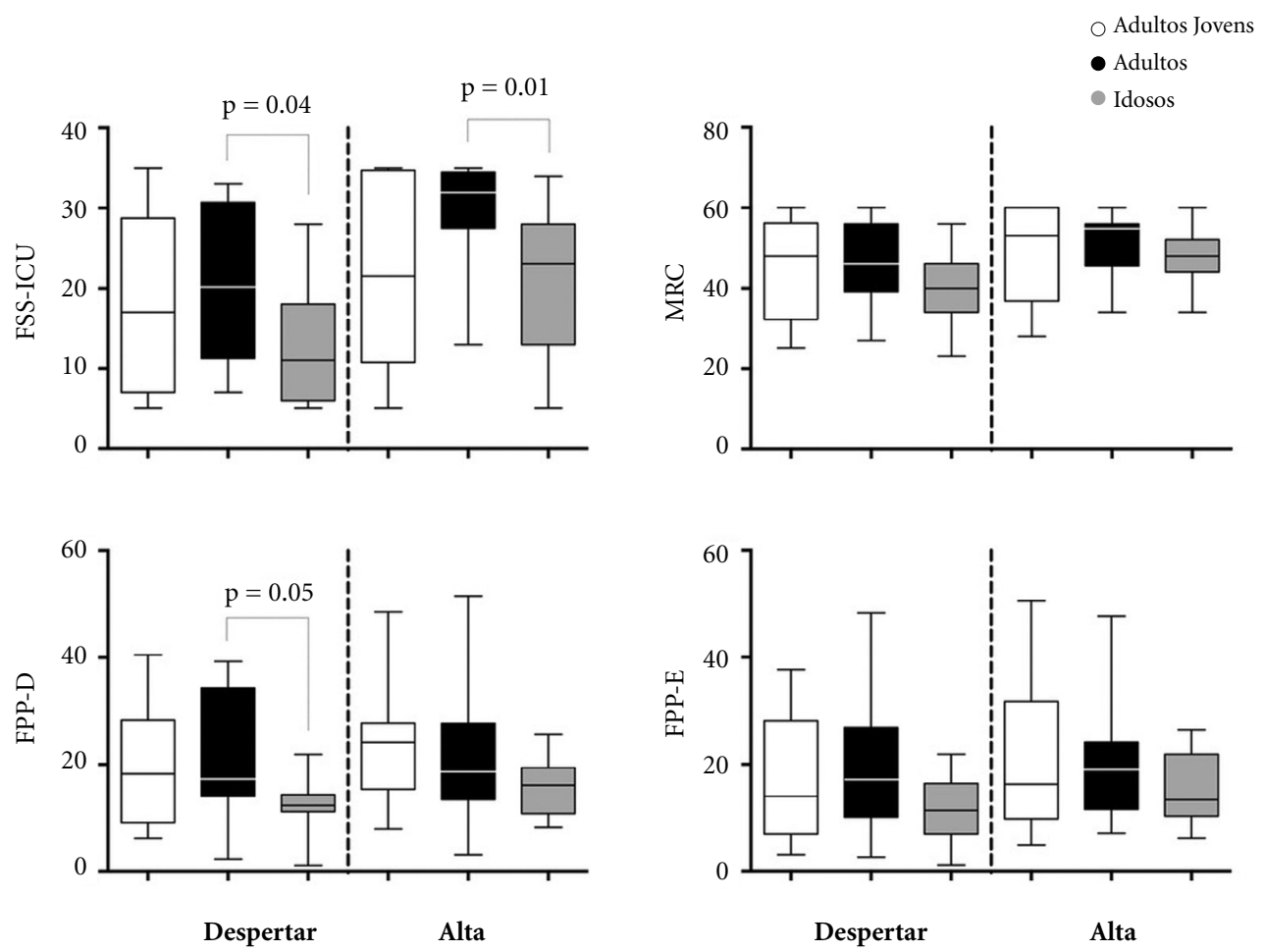

Legenda: FSS-ICU: Functional Status Score for the Intensive Care Unit; MRC-SS: Medical Research Council Sum-Score; FPP-D: Força de preensão palmar mão direta; FPP-E: Força de preensão palmar mão direta. Teste de Kruskal-Wallis. p-valor, nível de significância $p \leq 0.05$.

Figura 2. Análise do estado funcional e força muscular no momento do despertar e na alta da UTI entre os grupos adultos jovens, adultos e idosos.

Fonte: Elaborada pelos autores.

dade aspectos relacionados à resistência, força e virilidade. Dessa maneira, a fragilidade física e a ausência ao trabalho não provocam na mulher tanto desconforto como nos homens ${ }^{31}$.

A presença de comorbidades crônicas como hipertensão arterial (HAS) e a diabetes mellitus esteve presente em todas as faixas etárias, sendo mais prevalente nos idosos. Esse fato pode ser atribuído ao aumento da expectativa de vida nos últimos anos, impactando diretamente nas taxas de doenças crônicas, que são consideradas importantes fatores de risco cardiovascular, podendo elevar os desfechos circulatórios fatais ou não fatais, impactando nas taxas de internações dos idosos $^{32,33}$.

Em nosso estudo, a maior parte dos pacientes foram submetidos à ventilação mecânica, com os idosos com maior tempo de permanência. Estes achados são semelhantes aos descritos por Dietrich et al. ${ }^{34}$, que reportou uma média de $8,0 \pm 11,2$ dias no tempo de VM no grupo dos idosos, com idade entre 61-79 anos. O tempo de permanência na UTI identificado em nosso estudo foi superior a sete dias, e esse período observado em UTIs internacionais e brasileiras ${ }^{18,29,34,35}$, justificada pela gravidade e fisiopatologia clínica, que pode fazer com a permanência nesses setores dure de horas a semanas, conforme resposta clínica dos doentes críticos ao tratamento terapêutico empregado ${ }^{27}$.

Em idosos, o contexto da imobilidade em UTI, associado ao curso da doença e das alterações da própria senescência, pode resultar em importantes limitações que levam à perda da força da musculatura respiratória, tornando esse perfil de pacientes críticos mais vulneráveis ao desenvolvimento de complicações respiratórias, ao uso prolongado de VM, disfagias e broncoaspirações pós-extubação, impactando no aumento da mortalidade e custos hospitalares ${ }^{36}$. Segundo Yanping Ye et al. ${ }^{37}$ o custo médio diário 

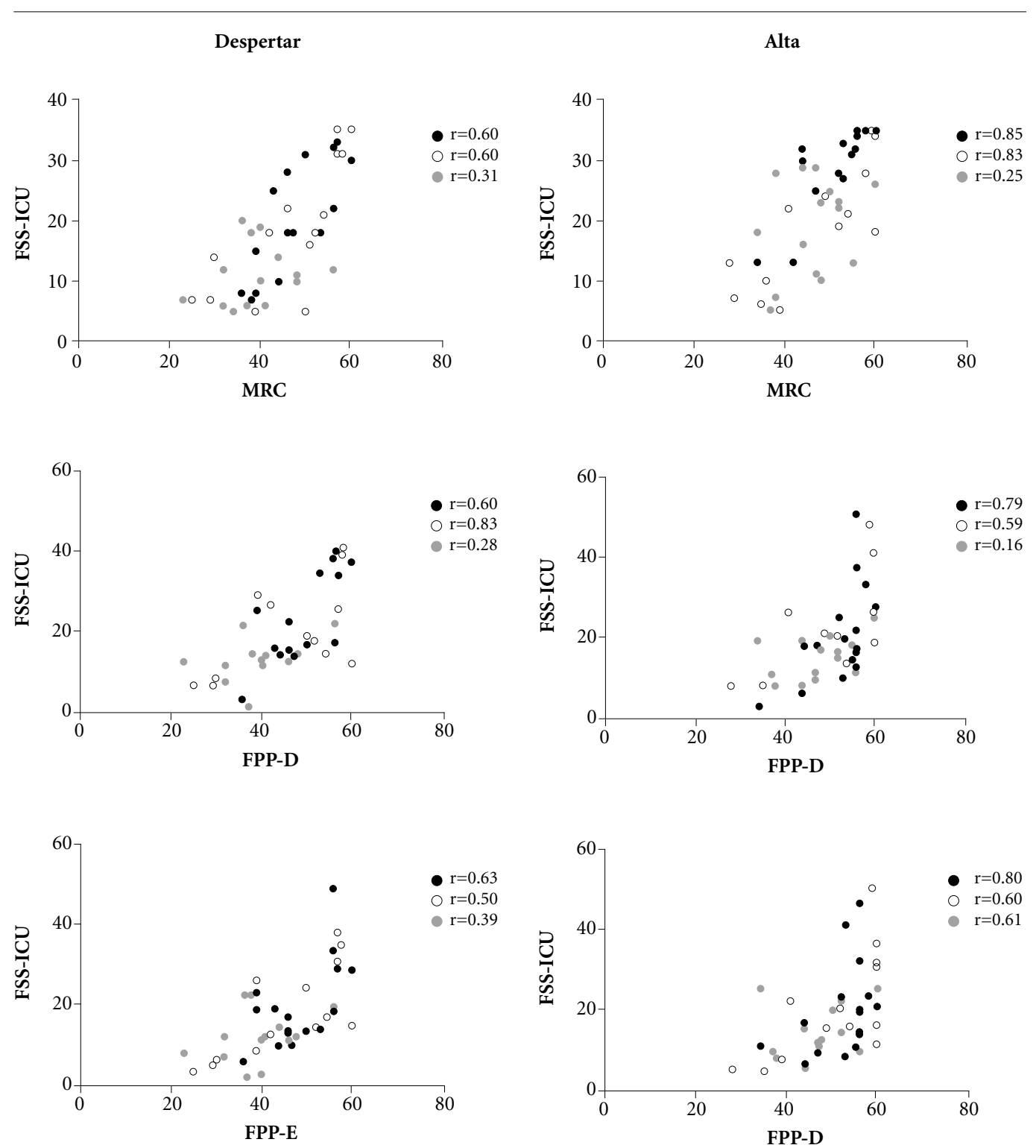

○ Adultos Jovens

- Adultos

Idosos

Legenda: FSS-ICU: Functional Status Score for the Intensive Care Unit; MRC-SS: Medical Research Council Sum-Score; FPP-D: Força de preensão palmar mão direta; FPP-E: Força de preensão palmar mão esquerda. Teste de correlação de Spearman.

Figura 3. Análise de Correlação entre a variável do estado funcional (FSS-ICU) e as variáveis de força muscular (MRC, FPP-D e FPP-E), nos diferentes grupos.

Fonte: Elaborada pelos autores.

de um paciente em UTI pode chegar a cerca de $\$ 1,212$ dólares. Portanto, por mais que o uso da VM seja necessário, se torna relevante a busca de estratégias para otimizar a sua duração a fim de minimizar possíveis complicações.
A FMA-UTI em nosso estudo foi prevalente nos idosos, sendo uma complicação frequente nessa faixa etária, e recorrente nos estágios agudos da doença ${ }^{38}$. As evidências atuais ${ }^{2,29,39-41}$ demonstram que a FMA-UTI reduz a funcionalidade e 
a mobilidade à beira leito e pode representar um preditor de baixo estado de saúde funcional até um ano após a alta da UTI. O estudo de Patel et al. ${ }^{42}$ demonstrou que tanto o aumento da idade como a gravidade da doença são fatores de risco para o desenvolvimento da FMA-UTI.

Nos pacientes criticamente enfermos, é eminente a redução da capacidade física, funcional e mental, quando comparada ao desempenho prévio a internação. Além disso, essas alterações podem ser potencializadas pelo tempo prolongado na UTI, uso de VM, sepse, disfunção múltiplas de órgãos, imobilidade no leito, uso sistêmico de corticoides, FMA-UTI, lenta resolução de lesão pulmonar e a idade ${ }^{11-14,16,26,28}$.

Observamos no despertar um estado funcional reduzido e um incremento gradativo desses parâmetros até a alta da UTI. Esses resultados foram semelhantes aos estudos que avaliaram doentes críticos por meio do FSS-ICU, assim como a força muscular avaliada pelo MRC-SS e dinamometria $^{14,15,43,44}$, apresentando um aumento expressivo na funcionalidade e força muscular durante a permanência do paciente no ambiente de UTI. O estudo de Zanni et al. ${ }^{14}$, demonstrou um aumento de 4 pontos no escore do FSS-ICU do momento basal até a alta da UTI. Similarmente, o estudo de Thrush et al, ${ }^{15}$ apresentou uma elevação de 5 pontos do escore acumulado do FSS-ICU. Adicionalmente, um estudo internacional ${ }^{21}$ de avaliação clinimétrica do FSS-ICU, envolvendo Estados Unidos, Austrália e o Brasil, demostraram uma boa magnitude do efeito $(2,02)$ do FSS-ICU desde o momento do despertar até a alta da UTI.

Em relação à força muscular, observamos um aumento progressivo durante a permanência do paciente na UTI, entretanto com uma evolução morosa, até atingir a condição muscular basal prévia $^{45}$. O recente estudo de Dietch et al. ${ }^{34}$ sistematizou uma amostra de idosos em UTI em dois grupos com faixa etária entre 61-79 anos e $\geq 80$ anos e identificaram a existência de diferenças no MRC-SS e na dinamometria no pós-alta imediato.

Nos idosos, o declínio do estado funcional foi mais acentuado, uma vez que a própria condição de senescência pode contribuir para essa redução. Assim, os fatores de risco eminentes na UTI, relacionados ao imobilismo, propiciam um desequilíbrio entre a síntese e degradação de proteínas musculares, associada à necrose das miofibrilas, proporcionando uma redução da massa muscular e da densidade mineral óssea ${ }^{46,47}$.
Entretanto, a estimativa de melhora nos idosos, em relação ao estado funcional e a força muscular foram expressivas, demonstrando um potencial de melhora dos indivíduos mais debilitados, justificando que esse perfil necessita de uma ação terapêutica clinicamente preventiva e intervencionista, relevantes para o alcance de um significativo efeito benéfico ${ }^{47}$. Portanto, as medidas preventivas e curativistas devem focar em estratégias da prática clínica, que impactam em características modificáveis, na busca por adoção de estratégias de abordagens individualizadas, direcionadas à manutenção ou ganho de força muscular global, mobilidade e funcionalidade de acordo com as demandas de cada perfil de paciente ${ }^{48}$.

O FSS-ICU apresentou uma correlação com as duas medidas de força muscular utilizadas no momento do despertar e na alta da UTI. Resultados semelhantes foram evidenciados na literatura $^{21,49}$ e apontaram associação do FSS-ICU com o MRC-SS, FPP, ICU mobility Scale, Activities of Daily Living (ADL) e Physical function in intensive care test scored (PFIT-s). Entretanto, os idosos apresentaram uma fraca associação em relação à funcionalidade e força muscular, provavelmente o declínio funcional do idosos não ocorre apenas por redução na força muscular, mas por ocasião de deficiências em múltiplos sistemas corporais, processos estes que podem estar relacionados às doenças diagnosticadas previamente ao período de internação ${ }^{50,51}$.

Este estudo possui limitações relacionadas ao conhecimento prévio à internação na UTI do estado funcional e força muscular, em razão da situação de urgência ocasionar dificuldades em avaliar tais informações, o que limitou a comparação das medidas obtidas com dados basais. Embora tenhamos alcançado o tamanho da amostra, pedimos cautelas para generalizar os dados para outras UTIs em virtude das características específicas da amostra estudada.

Dessa maneira, estudos observacionais e intervencionistas futuros em população idosa criticamente doente e em diferentes cenários de UTIs podem identificar outros fatores associados ao declínio funcional de idosos, que permitem nortear a intervenção precoce e específica para essa população fragilizada a fim de minimizar os danos da internação e do pós UTI, promovendo um retorno às atividade de vida diária de modo independente. 


\section{Conclusão}

O estado funcional e a força muscular apresentam progressiva melhora durante a permanência na UTI. Os idosos apresentam menor ganho do estado funcional, além de apresentarem menor força muscular desde o despertar. Estas variáveis apresentam importante associação nos adultos jovens e adultos, caracterizando uma a interação entre os diferentes domínios do estado funcional dos adultos. Nos idosos, as variáveis não estão correlacionadas dada a natureza multifatorial e multimorbidades do processo de adoecimento e incapacidade dos idosos.

\section{Colaboradores}

GS Martins participou da concepção do artigo, coletas, interpretação dos dados e redação do texto. SV Toledo contribuiu nas coletas. JML Andrade e EY Nakano realizaram análise estatística. LPS Paz auxiliou na análise estática e redação do texto. R Valduga participou da concepção do projeto. G Cipriano Júnior participou da revisão do texto. GFB Cipriano participou da concepção do projeto, artigo, revisão e aprovação da versão final a ser publicada.

\section{Agradecimentos}

Aos discentes de graduação do curso de Fisioterapia da Universidade de Brasília pelas contribuições nas coletas. A todos os voluntários e seus familiares que participaram deste estudo. $\mathrm{E}$ às instituições: Fundação de Apoio à Pesquisa do Distrito Federal (FAP/DF) e Conselho Nacional de Desenvolvimento Científico e Tecnológico (CNPq) pelo auxílio de fomento destinado ao projeto. E ao programa institucional de bolsas de mestrado da instituição de Coordenação de Aperfeiçoamento de Pessoal de Nível Superior (CAPES). 


\section{Referências}

1. Barbosa BR, Almeida JM, Barbosa MR, Rossi-Barbosa LAR. Avaliação da capacidade funcional dos idosos e fatores associados à incapacidade. Cien Saude Colet 2014; 19(8):3317-3325.

2. Hartley P, Costello P, Fenner R, Gibbins N, Quinn E, Kuhn I, Keevi VL, Romero-Ortuno R. Change in skeletal muscle associated with unplanned hospital admissions in adult patients: A systematic review and meta-analysis. PLoS ONE 2019; 14(1):1-21.

3. González-Seguel F, Corner EJ, Merino-Osorio C. International classification of functioning, disability, and health domains of 60 physical functioning measurement instruments used during the adult intensive care unit stay: a scoping review. Phys Ther 2019; 99(5):627-640.

4. Valenzuela PL, Javier S, Morales JS, Pareja-Galeano H, Izquierdo M, Enzo Emanuele E, Villa P, Lucia A. Physical strategies to prevent disuse-induced functional decline in the Elderly. Ageing Res Rev 2018; 47:8088 .

5. Pišot R, Marusic U, Biolo G, Mazzucco S, Lazzer S, Grassi B, Reggiani C, Toniolo L, di Prampero PE, Passaro A, NArici M, Mohammed S, Rittweger J, GAsparini M, Gabrijelcic BM, Simunic B. Greater loss in muscle mass and function but smaller metabolic alterations in older compared with younger men following $2 \mathrm{wk}$ of bed rest and recovery. J Appl Physiol 2016; 120(8):922-929.

6. Parry S, Puthucheary Z. The impact of extended bed rest on the musculoskeletal system in the critical care environment. Extrem Physiol Med 2015; 4:16.

7. Morten V, Jorgensen MG, Andreasen J, Rathleff MS, Molgaard CM. Very low levels of physical activity in older patients during hospitalization at an acute geriatric ward: a prospective cohort study. J Aging and Phys Act 2015; 23:542-549.

8. Tanner RE, Brunker LB, Agergaard J, Barrows KM, Briggs RA, Kwon OS, Young LM, Hopkins PN, Volpi E, Marcus RL, LAStayo PC, Drumond MJ. Age-related differences in lean mass, protein synthesis and skeletal muscle markers of proteolysis after bed rest and exercise rehabilitation. J Physiol 2015; 593:4259-4273.

9. Coker RH, Hays NP, Williams RH, Wolfe RR, Evans WJ. Bed rest promotes reductions in walking speed, functional parameters, and aerobic fitness in older, healthy adults. J Gerontol A Biol Sci Med Sci 2015; 70(1):91-96.

10. Doiron KA, Hoffmann TC, Beller EM. Early intervention (mobilization or active exercise) for critically ill adults in the intensive care unit. Cochrane Database Syst Rev 2018; 27(3):CD010754.

11. Welch C, Hassan-Smith ZK, Greig CA, Lord JM, Jackson TA. Acute sarcopenia secondary to hospitalisation - an emerging condition affecting older adults. Aging Dis 2018; 9(1):151-164.

12. Nydahl P, Sricharoenchai T, Chandra S Kundt FS, Huamg M, FischillM, Needham DM. Safety of patient mobilization and rehabilitation in the intensive care unit. Systematic review with meta-analysis. Ann Am Thorac Soc 2017; 14:766-777.
13. Van Ancum, Scheerman K, Jonkman NH, Smeenk HE, Kruizinga RC, Meskers CGM, Maier AB. Change in muscle strength and muscle mass in older hospitalized patients: A systematic review and meta-analysis. Exp Gerontol 2017; 92:34-41.

14. Zanni JM, Korupolu R, Fan E, Pradhan P, Janjua K, Palmer JB, Brower RG, Needham DM. Rehabilitation therapy and outcomes in acute respiratory failure: an observational pilot project. J Crit Care 2010; 25:254-262.

15. Thrush A, Rozek M, Dekerlegand JL. The clinical utility of the functional status score for the intensive care unit (FSS-ICU) at a long-term acute care hospital: a prospective cohort study. Phys Ther 2012; 92(12):1536-1545.

16. De Jonghe B, Sharshar T, Lefaucheur JP, Authier FJ, Durand-Zaleski I, Boussarsar M, Cerf C, Renaud E, Mesrati F, Carlet J, Raphaël JC, Outin H, BastujiGarin S. Paresis acquired in the intensive care unit: a prospective multicenter study. JAMA 2002; 288(2): 2859-2867.

17. De Jonghe B, Bastuji-Garin S, Durand MC, Malissin I, Rodrigues P, Cerf C, Outin H, Sharshar T. Respiratory weakness is associated with limb weakness and delayed weaning in critical illness. Crit Care Med 2007; 35(9):2007-2015.

18. Parry SM, Berney S, Granger CL, Dunlop DL, Murphy L, El-Ansary D, Koopman R, Denehy L. A new twotier strength assessment approach to the diagnosis of weakness in intensive care: an observational study. Crit Care 2015; 19(52):1-10.

19. Ali NA, O’Brien JM Jr, Hoffmann SP, Phillips G, Garland A, Finley JC, Almoosa K, Hejal R, Wolf KM, Lemeshow S, Connors AF Jr, Marsh CB; Midwest. Acquired weakness, handgrip strength, and mortality in critically ill Patients. Am J Respir Crit Care Med 2008; 178:261-268

20. Von Elm E, Altman DG, Egger M, Pocock SJ, Gotzche PC, Vandenbroucke JP. The strengthening the reporting of observational studies in epidemiology (STROBE) statement: guidelines for reporting observational studies. Bull World Health Organ 2007; 85:867-872.

21. Huang M, Chan KS, Zanni JM, Parry SM, Neto SG, Neto JA, da Silva VZ, Kho ME, Needham DM. Functional Status Score for the ICU: An international clinimetric analysis of validity, responsiveness, and minimal important difference. Crit Care Med 2016; 20(30):1-10.

22. Silva VZ, Neto JAA, Cipriano JG, Pinedo M, Needham DM, Zanni JM, Guimarães FSS. Versão brasileira da Escala de Estado Funcional em UTI: tradução e adaptação transcultural. Rev Bras Ter Intens 2017; 29(1):34-38.

23. Ciesla N, Dinglas V, Fan E, Kho M, Kuramoto J, Needham D. Manual muscle testing: a method of measuring extremity muscle strength applied to critically ill patients. J Vis Exp 2011; 12(50):2632.

24. Hermans G, Clerckx B, Vanhullebusch T, Segers J, Vanpee G, Robbeets C; Casaer MP, Wouters P, Gosselink R, Van Den Berghe G. interobserver agreement of medical research council sum- score and handgrip strength in the intensive care unit. Muscle Nerve 2012; 45:18-25. 
25. Tipping CJ, Harrold M, Holland A, Romero L, Nisbet T, Hodgson CL. The effects of active mobilisation and rehabilitation in ICU on mortality and function: a systematic review. Inten Care Med 2016; 43(2):171183.

26. Welch C, Hassan-Smith ZK, Greig CA, Lord JM, Jackson TA. Acute sarcopenia secondary to hospitalisation - an emerging condition affecting older adults. Aging Dis 2018; 9(1):151-164.

27. Parry SM, Granger CL, Berney S, Jones J, Beach L, ElAnsary D, Koopman R, Denehy L. Assessment of impairment and activity limitations in the critically ill: a systematic review of measurement instruments and their clinimetric properties. Intens Care Med 2015; 41(5):744-762.

28. Brauner YO, Adi N, Shahar BT, Yehezkel E, Carmeli E. Effect of physical therapy on muscle strength, respiratory muscles and functional parameters in patients with intensive care unit-acquired weakness. The Clin Respir J 2015; 9(1):1-6.

29. Tipping CJ, Bailey MJ, Bellomo R, Berney S, Buhr H, Denehy L, Harrold M, Holland A, Higgins AM, Iwashyna TJ, Needham D, Presneill J, Saxena M, Skinner EH, Webb S, Young P, Zanni J, Hodgson CL. The ICU mobility scale has construct and predictive validity and is responsive: a multicenter observational study. Ann Am Thorac Soc 2016; 13(6):887-893.

30. Costa JFM, Maia ACB. Concepções de homens hospitalizados sobre a relação entre gênero e saúde. Psic: Teor e Pesq 2009; 25(1):55-63.

31. Costa Junior, Couto MT, Maia ACB. Género y atención en la salud: puntos de vista de los profesionales que trabajan en el hospital y ambulatorio. Sex Salud Soc 2016; 23:97-117.

32. Costa AF, Flor MR, Campos MR, Oliveira AF, Costa MFS, Silva RS, Lobato LCP, Scramm JMA. Carga do diabetes mellitus tipo 2 no Brasil. Cad Saude Publica 2017; 33(2):1-14.

33. Lobo LA, Canuto R, Dias-da-Costa JS, Pattussi MP. Tendência temporal da prevalência de hipertensão arterial sistêmica no Brasil. Cad Saude Publica 2017; 33(6): 1-13.

34. Dietrich C, Cardoso JR,Vargas F, Sanchez EC, Dutra $\mathrm{FH}$, Moreira M. Capacidade funcional em idosos e idosos mais velhos após alta da unidade de terapia intensiva. Coorte prospectiva. Rev Bras Ter Intensiva 2017; 29(3):293-302.

35. Jesus FS, Paim DM, Brito JO, Barros IA, Nogueira TB, Prata B, Pires Q. Declínio da mobilidade dos pacientes internados em unidade de terapia intensiva. Rev Bras Ter Intensiva 2016; 28(2);114-119.

36. Stieff KV, Lim F, Chen L. Factors Influencing Weaning Older Adults from Mechanical Ventilation: An Integrative Review. Crit Care Nurs Q 2017; 40(2):165-177.

37. Yanping Ye, Bo Zhu, Li Jiang, Qi Jiang, Meiping Wang, Lin Hua, Xiuming Xi. A contemporary assessment of acute mechanical ventilation in Beijing: description, costs, and outcomes. Crit Care Med 2017;45(7):1160-1167.

38. Latronico N, Gosselink R. Abordagem dirigida para o diagnóstico de fraqueza muscular grave na unidade de terapia intensiva. Rev Bras Ter Intensiva 2015; 27(3):199-201.

39. Solverson KJ, Grant C, Doing CJ. Assessment and predictors of physical functioning post-hospital discharge in survivors of critical illness. Anm Intens Care 2016; 6(1):92.
40. Wieske L, Dettling-Ihnenfeldt D, Verhamme C, Nollet F, Schaik IN, Schultz M, Horn J, van der Schaaf M. Impact of ICU-acquired weakness on post-ICU physical functioning: a follow-up study. Critical Care 2015; 19:196.

41. Dettling-Ihnenfeldt D, Wieske L, Horn J, Nollet F, Van-der-Schaaf M. Functional Recovery in Patients with and Without Intensive Care Unit-Acquired Weakness. Amer J Phys Med Rehabil 2017; 96(4):297242.

42. Patel BK, Pohlman AS, Hall JB, Kress JP. Impact of Early Mobilization on Glycemic Control and ICU-Acquired Weakness in Critically Ill Patients Who Are Mechanically Ventilated. CHEST 2014; 146(3):583589.

43. Nordon-Craft A, Schenkman M, Edbrooke L, Malone DJ, Moss M, Denehy L. The physical function intensive care test: implementation in survivors of critical illness. Physical Therapy 2014; 94(10):1499-1507.

44. Silva PE, Maldaner V, Vieira L, Carvalho KL, Gomes H, Melo P, Babault N, Cipriano Jr, Durigan JLQ. Neuromuscular electrophysiological disorders and muscle atrophy in mechanically ventilated traumatic brain injury patients: new insights from a prospective observational study. J Crit Care 2018; 44:87-94.

45. Norman K, Stobäus N, Kulka K, Schulzke J. Effect of inflammation on handgrip strength in the non-critically ill is independent from age, gender and body composition. Eur J Clin Nut 2014; 68(2):155-158.

46. Corner EJ, Wood H, Englebretsen C, Thomas A, Grant RL, Nikoletou D, Soni N. The Chelsea Critical Care Physical Assessment Tool (CPAx): validation of an innovative new tool to measure physical morbidity in the general adult critical care population; an observational proof-of. concept pilot study. Physiotherapy 2013; 99(1):33-41.

47. Corner EJ, Wood H, Englebretsen C, Thomas A, Grant Parry S, Puthucheary Z. The impact of extended bed rest on the musculoskeletal system in the critical care environment. Extrem Physiol Med 2015; 4:16.

48. Peek K, Carey M, Sanson-Fisher R, Mackenzie L. Physiotherapists' perceptions of patient adherence to prescribed self-management strategies: a cross-sectional survey of Australian physiotherapists. Disabil Rehabil 2016; 39(19):1932-1938.

49. Parry SM, Denehy L, Beach LJ, Berney S, Williamson HC, Granger CL. Functional outcomes in ICU - what should we be using? - an observational study. Crit Care 2015; 29(19):127.

50. Denehy L, Lanphere J, Needham DL. Ten reasons why ICU patients should be mobilized early. Intens Care Med 2017; 43:86-90.

51. Cerri AP, Bellelli G, Mazzone A, Pittella F, Landi F, Zambon A, Annoni G. Sarcopenia and malnutrition in acutely ill hospitalized elderly: Prevalence and outcomes. Clin Nutr 2015; 34(4):745-751.

Artigo apresentado em 02/10/2018

Aprovado em 05/09/2019

Versão final apresentada em 07/09/2019

Editores-chefes: Romeu Gomes, Antônio Augusto Moura da Silva 